\title{
Chronic pain in multiple sclerosis: An overview
}

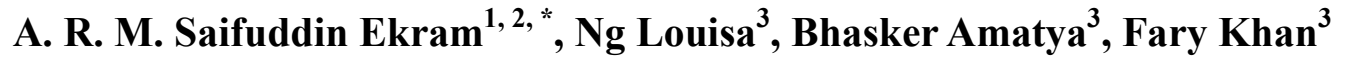 \\ ${ }^{1}$ Department of Medicine, Rajshahi Medical College, Rajshahi-6000, Bangladesh \\ ${ }^{2}$ Department of Epidemiology and Preventive Medicine, School of Public Health and Preventive Medicine, Monash University, Alfred \\ Hospital, Melbourne, VIC 3004, Australia \\ ${ }^{3}$ Department of Rehabilitation Medicine, Royal Melbourne Hospital, 34-54 Poplar Road Parkville, Melbourne, VIC 3052, Australia
}

\section{Email address:}

armsekram@yahoo.com (A. R. M. Saifuddin Ekram)

\section{To cite this article:}

A. R. M. Saifuddin Ekram, Ng Louisa, Bhasker Amatya, Fary Khan. Chronic Pain in Multiple Sclerosis: An Overview. American Journal of Internal Medicine. Vol. 2, No. 2, 2014, pp. 20-25. doi: 10.11648/j.ajim.20140202.13

\begin{abstract}
The pathophysiology of pain in multiple sclerosis (MS) is poorly understood, but there are multiple schools of thought. Different mechanisms are associated with causation of this pain e.g. acute pain due to inflammation; chronic or intermittent neuropathic pain related to central nervous system (CNS) lesions; pain secondary to spasticity, spasms and muscle cramps from higher motor neuron lesions; and musculoskeletal pain from adopting maladaptive body positions and general physical deconditioning. Similarly evidence for treating pain in MS is limited. Treatment is often based on anecdotal reports and clinical experience as there is scarcity of randomized placebo-controlled trials for evidence of pharmacological treatment of pain in MS. The aim of this article is to provide a brief review of current concepts about the nature, causes and management of pain in MS.
\end{abstract}

Keywords: Multiple Sclerosis, Chronic Pain, Neuropathic Pain

\section{Introduction}

Multiple sclerosis(MS) is primarily a chronic inflammatory disorder of the brain and spinal cord, characterized by episodes of neurological dysfunction due widespread microglial activation associated with extensive and chronic neurodegeneration, the clinical correlate of which is progressive accumulation of disability $[1,2]$. Worldwide incidence of multiple sclerosis is 3.6 per 100,000 person-years in women and 2 per 100,000 person-years in men and it affects 2.5 million people worldwide $[3,4]$.

It was once thought to be a "painless" disease[5]. But research has shown that chronic pain is a significant problem among persons with MS [6-11]. In this article we discussed in brief current concepts about the burden, nature, causes and management of pain in MS.

\subsection{Prevalence of Pain in MS}

Foley et al.[12] systematically identified prospective studies detailing pain prevalence in definite MS. They used pooled prevalence estimates, explored heterogeneity using meta-regression, and analyzed prevalence of the disease, using both estimates at disease milestones and longitudinal studies (Table 1).

\subsection{Types of Pain in MS}

Multiple mechanisms are associated with causation of pain in MS e.g. acute pain due to inflammation; chronic or intermittent neuropathic pain related to central nervous system (CNS) lesions; pain secondary to spasticity, spasms and muscle cramps from higher motor neuron lesions; and musculoskeletal pain from adopting maladaptive body positions and general physical deconditioning [13]. But Truini et al. [14] suggested that pain might be more effectively classified and treated according to symptoms and underlying mechanisms (Table 2). The new mechanismbased classification proposed distinguishes following types of MS-related pain:

\subsubsection{Continuous Central Neuropathic Pain}

Central pain is an important symptom in MS (around $30 \%$ ) and causes much suffering [15]. It is described as a continuous, aching, pricking or burning pain that is typically bilateral and worse at night, predominantly affecting the legs and feet. It may also be exacerbated by physical activity[16]. The pain is intense with small to moderate spontaneous variation. Central neuropathic pain (CNP) is believed to be a consequence of damage to myelinated nerves in the CNS, and can be propagated by 
two mechanisms:

1. Generation of ectopic impulses at demyelinated lesions in response to neural damage[17].

2. Interruption of inhibitory impulses from the brain, which removes the modulation of afferent A-delta and C pain pathways.

The absence of inhibitory impulses means that stimuli from peripheral receptors, which are normally below the threshold to generate pain, can now register pain.

\subsubsection{Intermittent Central Neuropathic Pain}

- Lhermitte's phenomenon

Some patients with MS have a transient, short-lasting sensation at the back of the neck, lower back or other parts of the body following forward bending of the neck. The sensation, known as Lhermitte's phenomenon, is usually electric-like but can be tingling, buzzing or otherwise. It propagates rapidly and disappears on resuming normal posture. It is caused by demyelination in the cervical spinal cord due to MS, but can also occur in other cervical spine disorders (such as demyelination of posterior columns from cisplatin therapy, pernicious anemia or from cervical herpes zoster) [18].

- Trigeminal neuralgia

In general population trigeminal neuralgia (TN) mainly affects people over 60 . Among people over 60 , it has a prevalence of $0.1-0.2$ per thousand and an incidence ranging from about $4-5 / 100$ 000/year to $20 / 100$ $000 /$ year[19]. Lifetime prevalence of TN was estimated to be $0.3 \%$ [ 10 of $3336 ; 95 \%$ CI $0.1-0.5 \%$ ] [20]. Among MS cases, its prevalence was $2 \%$ to $6.3 \%[9,21]$. TN leads to paroxysms of short-lasting but very severe pain. Between attacks, patients are usually asymptomatic, but a constant dull background pain persists in some cases [22]. MSrelated TN is more likely to be bilateral and they tend to be younger than non-MS TN patients [16].

\subsubsection{Musculoskeletal Pain}

Musculoskeletal pain is common in MS. It is thought to be aggravated by abnormal gait, abnormal posture, prolonged immobility and wheelchair use [17].

- Low back pain

The prevalence of back pain in people with MS has been reported to be between $14 \%$ and 39\% [23-25]. Some authors have suggested that back pain and burning in the extremities are the most common complaints reported by this population $[23,26]$. Chronic low back pain is most commonly due to abnormal stresses on the paravertebral muscles resulting in degenerative changes and facet joint problems, in addition to the effects of spasticity, bad posture and improper seating[17, 26, 27]. We should not forget that it may be caused by underlying conditions such as osteoporosis and demyelinating lesion in the spinal cord [26].

\section{- $\quad$ Painful tonic spasms}

Painful tonic spasms are caused by spontaneous discharges from damaged axons spreading to adjacent nerves. They are associated with demyelinating lesions in the basal ganglia, cerebral peduncle, internal capsule, medulla and spinal cord. They may be triggered by touch, movement, hyperventilation or emotions and can occur several times per day. Each episode lasts less than 2 minutes $[16,28]$. Patients with pain were significantly more often spastic[10].

\subsubsection{Mixed Neuropathic and Non-Neuropathic Pain}

- Headache

MS can cause headaches[29]. The association between migraine and MS is biologically plausible and has been confirmed by general studies[30]. Migraine is three-times more common in MS patients than in the general population; and the MS-migraine group was more symptomatic than the MS-no headache group[31]. Lifetime prevalence of any type of headache is estimated to be $35.5 \% ; 21.4 \%$ of MS patients have had a tensive headache, $16 \%$ have suffered from migraine and $1.9 \%$ has had both. Among MS patients suffering from migraine, 9.2\% had aura, while $90.8 \%$ did not [32].

\section{Methods of Treatment of Pain in Multiple Sclerosis}

In general, the evidential basis for choosing how to treat pain in MS is limited. Treatment is often based on anecdotal reports and clinical experience because randomized placebo-controlled trials (RCTs) concerning pharmacological treatment of pain in MS are scarce [14, 33]. Most of these RCTs did not collect detailed information about the type and intensity of pain, but only collected a small amount of information as part of assessing quality of life or spasticity. Many trials assessed cannabis derivatives, but there is lack of documented RCT on the drugs that have for long been most popular in treating neuropathic pain (amitriptyline and carbamazepine) or are currently popular (pregabalin and serotonin noradrenaline reuptake inhibitors)[34, 35].

\subsection{Treatment of Painful Tonic Spasms (PTS)}

Treatments of PTS are variable. Anecdotal medications such as baclofen, benzodiazepines, gabapentin and carbamazepine are used. In open-label studies gabapentin and tiagabine were reported to be effective $[36,37]$. In another open-label study, botulinum toxin was found to be effective for PTS [38]. PTS is treated with these anecdotal drugs, but definitive evidence is still lacking.

\subsection{Treatment of Low Back Pain}

The use of NSAIDs or opioids for treating back pain in patients with MS does not differ from the treatment of low back pain in the general population [17,33].

\subsection{Treatment of Neuropathic Pain}

- Tricyclic antidepressants Amitriptyline, nortriptyline and clomipramine are 
commonly-used tricyclic antidepressants for the treatment of central neuropathic pain in MS. Drowsiness, constipation, urinary retention and hypotension are common adverse effects. Though commonly used, the evidence for optimal dosing regimens has not been evaluated in randomized clinical trials of patients with MS[33].

- Antiepileptic medications

Carbamazepine, gabapentin, pregabalin, levetiracetam and lamotrigine are frequently used in pain management. But adverse effects accompany these medications and this may limit their use in many patients who are unable to tolerate them at the doses required to reach efficacy. Antiepileptic medications may be appropriate in some people with MS pain, but the evidential basis for this treatment is weak, due to the small numbers and uncontrolled designs[33].

\section{- Intrathecal baclofen}

Intrathecal baclofen is used in patients with MS with spinal lesions. But the evidence is weak to support the administration of intrathecal therapy and its use should be carefully determined on an individual basis when oral therapy is not tolerated or is ineffective[33].

- Opioid analgesics

Opioid analgesics are used for relief of pain in patients with MS. Their role in neuropathic pain is controversial. The literature is scarce on the effects of oral morphine and tramadol for the treatment of neuropathic pain in MS[33].

\section{- Anaesthetic and Antiarrhythmic Agents}

Lidocaine and mexilletine are used in tonic seizures and paroxysmal pain. There have been good results in some cases, but this should be regarded as only observational evidences[33].

\section{- Cannabinoids}

Cannabinoids have been among the few pain treatments studied in well designed, randomized, placebo-controlled trials. Because pain in MS is quite heterogeneous, additional studies of cannabinoids should differentiate the origins of pain syndromes in order to help identify cannabinoid responders. Evidence confirms that the frequency and severity of adverse events with the use of cannabinoids is significant. So it must be taken into consideration if cannabinoids are to ever become a mainstream therapeutic option for neuropathic pain in MS[33].

\subsection{Treatment of Trigeminal Neuralgia}

Treatment of trigeminal neuralgia in MS is based on studies of idiopathic $\mathrm{TN}$ and usually consists of antiepileptic medications such as carbamazepine and lamotrigine [33, 39]. Gabapentin, topiramate and prostaglandin E1 analogue misoprostol are reported to be effective in some open label studies [40-45]. For patients who are refractory to medical therapy, Gasserian ganglion percutaneous techniques, gamma-knife surgery and microvascular decompression are the most promising invasive treatment options[22]. The evidential basis is inadequate as the majority of studies on medications for trigeminal neuralgia are done on open-label design. Inadequacy of sample size and the inability to perform placebo-controlled studies due to ethical considerations further limit our ability to provide evidences for optimal treatment of trigeminal neuralgia.

\subsection{Treatment of Lhermitte's Phenomenon}

Most subjects who experience Lhermitte's phenomenon do not seek treatment. A low dose of carbamazepine is used in persistent cases to reduce frequency and severity [18, 33].

\subsection{Non-Pharmacological Treatment of Pain in MS}

There is no consensus in the medical community regarding which therapies are considered alternative, complementary, or unconventional[46]. However, complementary and alternative medicine has been defined as "unconventional medicine" [47] and the term generally refers to forms of treatment not taught widely in medical schools or not typically available in hospitals [48, 49]. Three types of non-disease-modifying agents (non-DMA) are used by patients with multiple sclerosis: (1) dietary supplements (e.g. vitamins and minerals); (2) exercise modalities, including yoga; and (3) therapies that may be considered "true" complementary and alternative medicine (CAM), such as acupuncture, chiropractic, and massage therapy[46]. Along with these we can consider mainstream treatments and therapies for patients with multiple sclerosis, such as osteopathic manipulative treatment (OMT), physical therapy and behavioral therapy.

CAM has become popular for the management of symptoms among people with MS [50, 51]. A survey [52] indicated that $50-75 \%$ of MS patients use CAM, often reporting improvement in function, decreased symptom severity and dissatisfaction with conventional treatment [50, 53]. Indeed, $66 \%$ of participants in another survey[51] reported improvements in their symptoms following CAM. However, in spite of the popularity of these therapies, little scientific evidence exists as to their benefits $[50,51]$. Most importantly these studies did not look at about relief of pain in MS [26]. Tai Chi, meditation and hypnotherapy may serve to increase quality of life and reduce pain in MS[54]. Several studies have reported positive effects of reflexology in the treatment of pain; however, there is scarcity of randomized controlled clinical trials for the treatment of pain in MS. One RCT reported that precision reflexology was not superior to sham treatment. However, both treatments achieved clinically significant improvements for MS symptoms via a possible placebo effect or stimulation of reflex points in the feet using non-specific massage [26]. A quasi-experimental trial comparing the effects of selfhypnosis training (HYP) with progressive muscle relaxation (PMR) showed reduction in pain intensity and pain interference in MS subjects. HYP-condition participants reported significantly greater decreases in pain and pain interference than PMR-condition participants, and 
gains were maintained at 3-month follow-up [49]. But there has been no RCT on hypnosis in MS pain. A pilot study has demonstrated that transcutaneous electrical nerve stimulation has clinical relevance as a non-pharmacological method of pain relief for low back pain in people with MS [55]. But again in this field no RCT is available.

\section{Role of Rehabilitation}

Improved understanding of pain prevalence, chronic pain-related disability and impact on quality of life in persons with MS and early intervention may contribute to the overall well-being and this will decrease pain related disability in this population[56]. A recent study revealed that patient with longer duration of MS, reported higher disability, severely limiting their daily activities due to pain [57]. A multidisciplinary rehabilitation approach with access to specialized chronic pain management clinics can minimize pain-related disability in this group. More emphasis should be given to spasticity management, psychological and psychosocial support and factors impacting chronic pain contributing to limitation in activity and participation[57].

Table 1. Pain prevalence in MS[12]

\begin{tabular}{lll}
\hline & Quantified prevalence & $\mathbf{9 5 \%}$ CI \\
\hline Overall pain prevalence & $63 \%$ & $55-70 \%$ \\
Headache & $43 \%$ & $33-52 \%$ \\
Neuropathic extremity pain & $26 \%$ & $7-53 \%$ \\
Back pain & $20 \%$ & $13-28 \%$ \\
Painful spasms & $15 \%$ & $8.5-23 \%$ \\
Lhermitte's sign & $16 \%$ & $10-25 \%$ \\
Trigeminal neuralgia & $3.8 \%$ & $2-6 \%$ \\
\hline
\end{tabular}

Table 2. Types of pain in MS

\begin{tabular}{|c|c|}
\hline $\begin{array}{l}\text { Pain type as classified by } \\
\text { Truini et al.[14] }\end{array}$ & $\begin{array}{l}\text { Pain type as classified by } \\
\text { O'Connor et al.[13] }\end{array}$ \\
\hline \multicolumn{2}{|l|}{ Neuropathic pain } \\
\hline $\begin{array}{l}\text { Ongoing extremity pain (12- } \\
28 \%)\end{array}$ & Continuous central neuropathic pain \\
\hline Trigeminal neuralgia $(2-5 \%)$ & Intermittent central neuropathic pain \\
\hline Lhermitte's phenomenon $(15 \%)$ & Intermittent central neuropathic pain \\
\hline Painful tonic spasms $(6-11 \%)$ & Musculoskeletal pain \\
\hline Spasticity pain $(50 \%)$ & Musculoskeletal pain \\
\hline \multicolumn{2}{|l|}{ Nociceptive pain } \\
\hline \multicolumn{2}{|c|}{ Nerve trunk pain associated with optic neuritis $(8 \%)$} \\
\hline \multicolumn{2}{|c|}{ Musculoskeletal pains induced by postural anomalies } \\
\hline Back pain $(10-16 \%)$ & Musculoskeletal pain \\
\hline Migraine (34 \%) & $\begin{array}{l}\text { Mixed neuropathic and non- } \\
\text { neuropathic pain }\end{array}$ \\
\hline Tension-type headache $(21 \%)$ & $\begin{array}{l}\text { Mixed neuropathic and non- } \\
\text { neuropathic pain }\end{array}$ \\
\hline $\begin{array}{l}\text { Treatment-induced pains } \\
\text { Other pains }\end{array}$ & \\
\hline
\end{tabular}

\section{Conclusion}

Pain in multiple sclerosis is an often-overlooked and poorly-treated complication of this common cause of neurological disability. Treatment of MS patients' pain should be specific for each type of pain - nociceptive or neuropathic - and should be based on a multidisciplinary multimodal approach to best improve patient outcomes. The evidential basis for treatment of chronic pain in MS should be strengthened.

\section{Acknowledgement}

We express our gratitude to Geoffrey Keith Robinson for his help in grammatical correction of the manuscript.

\section{References}

[1] Compston, A. and A. Coles, Multiple sclerosis. Lancet, 2002. 359(9313): p. 1221-31.

[2] Frohman, E.M., et al., The utility of MRI in suspected MS: report of the Therapeutics and Technology Assessment Subcommittee of the American Academy of Neurology. Neurology, 2003. 61(5): p. 602-11.

[3] Alonso, A. and M.A. Hernan, Temporal trends in the incidence of multiple sclerosis: a systematic review. Neurology, 2008. 71(2): p. 129-35.

[4] Programme for Neurological Disease and Neurosciences Department of Mental Health and Substance Abuse, Atlas: Country Resources for Neurological Disorders: Results of a Collaborative Study of World Health Organization and World Federation of Neurology, World Health Organization, Geneva, Switzerland, 2004.

[5] Aring, C.D., Pain in multiple sclerosis. JAMA, 1973. 223(5): p. 547.

[6] Archibald, C.J., et al., Pain prevalence, severity and impact in a clinic sample of multiple sclerosis patients. Pain, 1994. 58(1): p. 89-93.

[7] Svendsen, K.B., et al., Sensory function and quality of life in patients with multiple sclerosis and pain. Pain, 2005. 114(3): p. 473-81.

[8] Beiske, A.G., et al., Pain and sensory complaints in multiple sclerosis. Eur J Neurol, 2004. 11(7): p. 479-82.

[9] Solaro, C., et al., The prevalence of pain in multiple sclerosis: a multicenter cross-sectional study. Neurology, 2004. 63(5): p. 919-21.

[10] Stenager, E., L. Knudsen, and K. Jensen, Acute and chronic pain syndromes in multiple sclerosis. Acta Neurol Scand, 1991. 84(3): p. 197-200.

[11] Vermote, R., P. Ketelaer, and H. Carton, Pain in multiple sclerosis patients. A prospective study using the Mc Gill Pain Questionnaire. Clin Neurol Neurosurg, 1986. 88(2): p. 87-93.

[12] Foley, P.L., et al., Prevalence and natural history of pain in adults with multiple sclerosis: Systematic review and metaanalysis. Pain, 2012.

[13] O'Connor, A.B., et al., Pain associated with multiple sclerosis: systematic review and proposed classification. Pain, 2008. 137(1): p. 96-111.

[14] Truini, A., et al., "A mechanism-based classification of pain in multiple sclerosis." J Neurol, 2013. 260(2): 351-367. 
[15] Osterberg, A., J. Boivie, and K.A. Thuomas, Central pain in multiple sclerosis--prevalence and clinical characteristics. Eur J Pain, 2005. 9(5): p. 531-42.

[16] Lalkhen, A.G., J.P. Bedford, and A.D. Dwyer, Pain associated with multiple sclerosis: epidemiology, classification and management. British Journal of Neuroscience Nursing, 2012. 8(5): p. 267-74.

[17] Moulin, D.E., Pain in central and peripheral demyelinating disorders. Neurol Clin, 1998. 16(4): p. 889-98.

[18] Al-Araji, A.H. and J. Oger, Reappraisal of Lhermitte's sign in multiple sclerosis. Mult Scler, 2005. 11(4): p. 398-402.

[19] Manzoni, G.C. and P. Torelli, Epidemiology of typical and atypical craniofacial neuralgias. Neurol Sci, 2005. 26 Suppl 2: p. s65-7.

[20] Mueller, D., et al., Prevalence of trigeminal neuralgia and persistent idiopathic facial pain: a population-based study. Cephalalgia, 2011. 31(15): p. 1542-8.

[21] Putzki, N., et al., Prevalence of migraine, tension-type headache and trigeminal neuralgia in multiple sclerosis. Eur J Neurol, 2009. 16(2): p. 262-7.

[22] Obermann, M. and Z. Katsarava, Update on trigeminal neuralgia. Expert Rev Neurother, 2009. 9(3): p. 323-9.

[23] Clifford, D.B. and J.L. Trotter, Pain in multiple sclerosis. Arch Neurol, 1984. 41(12): p. 1270-2.

[24] Moulin, D.E., Pain in multiple sclerosis. Neurol Clin, 1989. $7(2)$ : p. $321-31$

[25] Kassirer, M.R. and D.H. Osterberg, Pain in chronic multiple sclerosis. J Pain Symptom Manage, 1987. 2(2): p. 95-7.

[26] Maloni, H.W., Pain in multiple sclerosis: an overview of its nature and management. J Neurosci Nurs, 2000. 32(3): p. $139-44,152$.

[27] Thompson, A.J., Multiple sclerosis: symptomatic treatment. J Neurol, 1996. 243(8): p. 559-65.

[28] Matthews, W.B., Paroxysmal symptoms in multiple sclerosis. J Neurol Neurosurg Psychiatry, 1975. 38(6): p. 617-23.

[29] Rolak, L.A. and S. Brown, Headaches and multiple sclerosis a clinical study and review of the literature. J Neurol, 1990. 237(5): p. 300-2.

[30] Kister, I., et al., Tension-type headache and migraine in multiple sclerosis. Curr Pain Headache Rep, 2010. 14(6): p. $441-8$

[31] Kister, I., et al., Migraine is comorbid with multiple sclerosis and associated with a more symptomatic MS course. J Headache Pain, 2010. 11(5): p. 417-25.

[32] Martinelli Boneschi, F., et al., Lifetime and actual prevalence of pain and headache in multiple sclerosis. Mult Scler, 2008. 14(4): p. 514-21.

[33] Solaro, C. and M. Messmer Uccelli, Pharmacological management of pain in patients with multiple sclerosis. Drugs, 2010. 70(10): p. 1245-54.

[34] Attal, N., et al., EFNS guidelines on the pharmacological treatment of neuropathic pain: 2010 revision. Eur J Neurol, 2010. 17(9): p. 1113-e88.
[35] Tan, T., et al., Pharmacological management of neuropathic pain in non-specialist settings: summary of NICE guidance. Bmj, 2010. 340: p. c1079.

[36] Solaro, C., et al., Gabapentin is effective in treating nocturnal painful spasms in multiple sclerosis. Mult Scler, 2000. 6(3): p. 192-3.

[37] Restivo, D.A., et al., Botulinum toxin treatment of painful tonic spasms in multiple sclerosis. Neurology, 2003. 61(5): p. 719-20.

[38] Al-Smadi, J., et al., A pilot investigation of the hypoalgesic effects of transcutaneous electrical nerve stimulation upon low back pain in people with multiple sclerosis. Clin Rehabil, 2003. 17(7): p. 742-9.

[39] Espir, M.L. and P. Millac, Treatment of paroxysmal disorders in multiple sclerosis with carbamazepine (Tegretol). J Neurol Neurosurg Psychiatry, 1970. 33(4): p. 528-31.

[40] Khan, O.A., Gabapentin relieves trigeminal neuralgia in multiple sclerosis patients. Neurology, 1998. 51(2): p. 611-4.

[41] Solaro, C., et al., An open-label trial of gabapentin treatment of paroxysmal symptoms in multiple sclerosis patients. Neurology, 1998. 51(2): p. 609-11.

[42] Zvartau-Hind, M., et al., Topiramate relieves refractory trigeminal neuralgia in MS patients. Neurology, 2000. 55(10): p. 1587-8.

[43] Solaro, C., et al., Topiramate relieves idiopathic and symptomatic trigeminal neuralgia. J Pain Symptom Manage, 2001. 21(5): p. 367-8.

[44] Reder, A.T. and B.G. Arnason, Trigeminal neuralgia in multiple sclerosis relieved by a prostaglandin $\mathrm{E}$ analogue. Neurology, 1995. 45(6): p. 1097-100.

[45] Misoprostol in the treatment of trigeminal neuralgia associated with multiple sclerosis. J Neurol, 2003. 250(5): p. $542-5$.

[46] Bowling, A.C. and T.M. Stewart, Current Complementary and Alternative Therapies for Multiple Sclerosis. Curr Treat Options Neurol, 2003. 5(1): p. 55-68.

[47] Eisenberg, D.M., et al., Unconventional medicine in the United States. Prevalence, costs, and patterns of use. N Engl J Med, 1993. 328(4): p. 246-52.

[48] Eisenberg, D.M., et al., Trends in alternative medicine use in the United States, 1990-1997: results of a follow-up national survey. Jama, 1998. 280(18): p. 1569-75.

[49] Jensen, M.P., et al., A comparison of self-hypnosis versus progressive muscle relaxation in patients with multiple sclerosis and chronic pain. Int J Clin Exp Hypn, 2009. 57(2): p. $198-221$

[50] Apel, A., B. Greim, and U.K. Zettl, How frequently do patients with multiple sclerosis use complementary and alternative medicine? Complement Ther Med, 2005. 13(4): p. 258-63.

[51] Murray, T.J., Complementary and alternative medicine for MS. Int MS J, 2006. 13(1): p. 3

[52] Carlson, M.J. and G. Krahn, Use of complementary and alternative medicine practitioners by people with physical disabilities: estimates from a National US Survey. Disabil Rehabil, 2006. 28(8): p. 505-13. 
[53] Hughes, C.M., S. Smyth, and A.S. Lowe-Strong, Reflexology for the treatment of pain in people with multiple sclerosis: a double-blind randomised shamcontrolled clinical trial. Mult Scler, 2009. 15(11): p. 1329-38.

[54] Huntley, A. and E. Ernst, Complementary and alternative therapies for treating multiple sclerosis symptoms: a systematic review. Complement Ther Med, 2000. 8(2): p. $97-105$.

[55] Nayak, S., et al., Use of unconventional therapies by individuals with multiple sclerosis. Clin Rehabil, 2003. 17(2): p. 181-91.

[56] Khan, F. and J. Pallant, Chronic pain in multiple sclerosis: prevalence, characteristics, and impact on quality of life in an Australian community cohort. J Pain, 2007. 8(8): p. 61423.

[57] Khan, F., B. Amatya, and J. Kesselring, Longitudinal 7-year follow-up of chronic pain in persons with multiple sclerosis in the community. J Neurol, 2013. 260(8): p. 2005-15. 